\title{
FIB TEM Sample Preparation of Deeply Buried Interfaces
}

\author{
M. J. Rye ${ }^{1}$, J. R. Michael ${ }^{1}$, and N. Y. C. Yang ${ }^{2}$ \\ ${ }^{1}$ Sandia National Laboratories, PO Box 5800, Albuquerque, NM 87185-0886 \\ ${ }^{2}$ Sandia National Laboratories, PO Box 969, Livermore, CA 94551
}

Quality sample preparation is an extremely important and sometimes difficult part of TEM analysis. Dual platform focused ion beam tools (both SEM and FIB columns on one chamber) have provided unique ways to produce samples for TEM. However, there are some sample geometries that are challenging and require novel approaches to TEM sample preparation with the FIB. Typical FIB sample preparation results in a thin sample that is about $20 \mu \mathrm{m}$ wide by $10 \mu \mathrm{m}$ deep. TEM sample preparation with FIB of structures that are buried deeper than $10 \mu \mathrm{m}$ can be difficult without some mechanical removal of material that may not be exacting enough to expose the layer of interest. FIB preparation for TEM of interfaces that are deeply buried in a sample are particularly challenging. Here we propose a method that allows deeply buried layers or interfaces to be exposed and quality TEM thin samples prepared.

The sample of interest in this case was BiTe with a thick Au plating $(15 \mu \mathrm{m})$. The interface between the BiTe substrate and the Au plating was the area of interest. In this case, because of the depth of the interface traditional techniques used for TEM sample preparation in the FIB were not adequate. The instrument used was a FEI dual platform Helios NanoLab 600 FIB. The procedure involves the removal of most of the Au plating by tilting the sample $-10^{\circ}$ with respect to the ion beam and then a large beam current is used to remove an area of the coating that is about $35 \mu \mathrm{m}$ wide to allow adequate area for working. For increased speed this is done by using a "cleaning" cross section type cut where the beam is rastered in a line rather than in a rectangle. This is shown in Fig.1 .Once this cut is complete, the sample is rotated $180^{\circ}$ and the cut is repeated as shown in Fig. 2. The goal of this step is to leave a thin Au-layer on top of the BiTe substrate. At this point a standard TEM sample is produced at the bottom of the two cuts where the Au-layer is thin as shown in Fig. 3. From this point on prepare a normal ex-situ liftout sample as shown in Fig. 4 and the completed sample in Fig. 5. See the TEM image of completed sample in Fig 6.

Previous to using this technique, we employed mechanical methods to remove the bulk of the surface layer before FIB sample preparation for the TEM was attempted. The technique discussed here will not work for all samples with deeply buried layers as there is a limit to how much material can be removed with the FIB and if the layers exceed $30 \mu \mathrm{m}$. In that case, a combination of mechanical removal of the layer and the FIB technique discussed here would be utilized. This preparation technique can also be used for in-situ preparation and removal of TEM samples as well as cross sections for SEM imaging and EDS analysis.

[1] Sandia National Laboratories is a multi-program laboratory operated by Sandia Corporation, a wholly owned subsidiary of Lockheed Martin Company, for the U.S. Department of Energy's National Nuclear Security Administration under contract DE-AC04-94AL85000. 


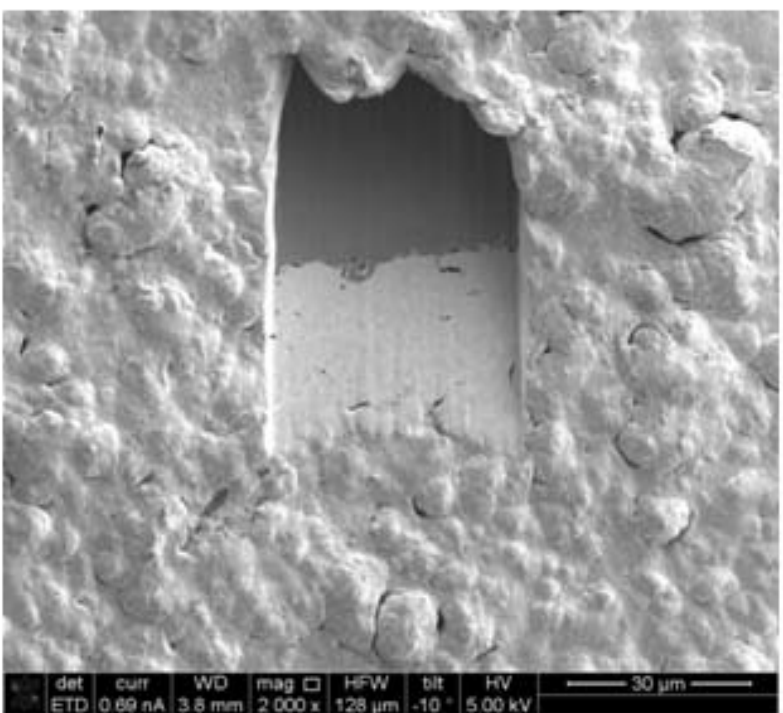

Figure1. SEM image of initial surface cut at low angle $\quad(-10$ deg Stage tilt). Milledusing the cleaning $x$-section tool and cut until the buried interface was revealed

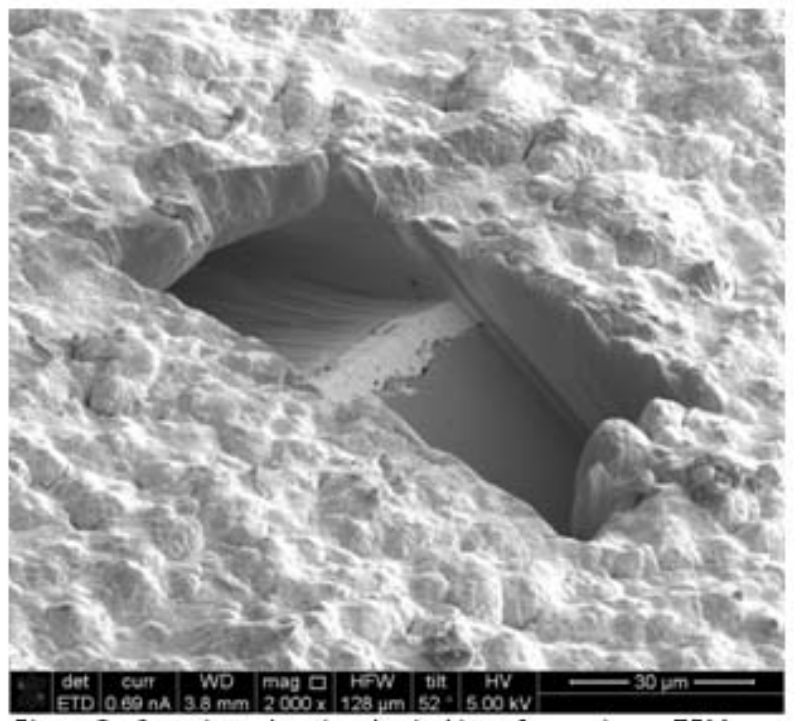

Figure 3. Overview showing buried interface prior to TEM sample preparation

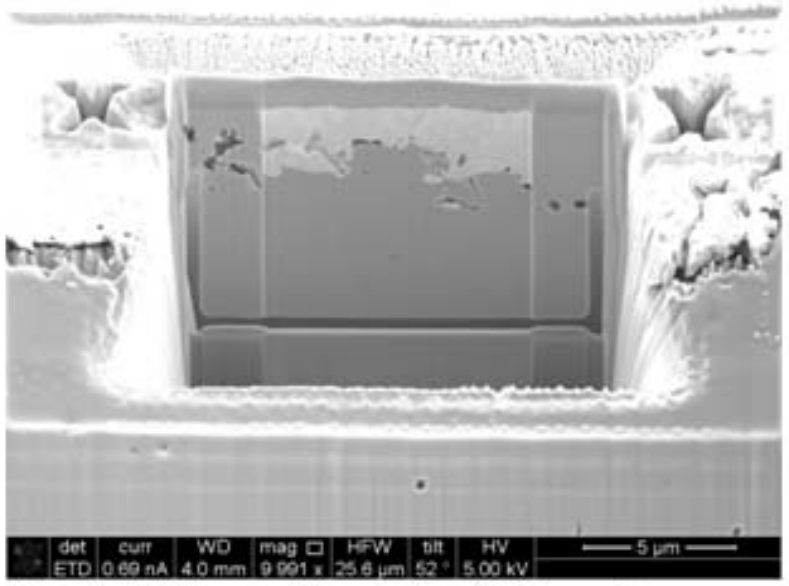

Figure 5. SEM image of the completed TEM x-section

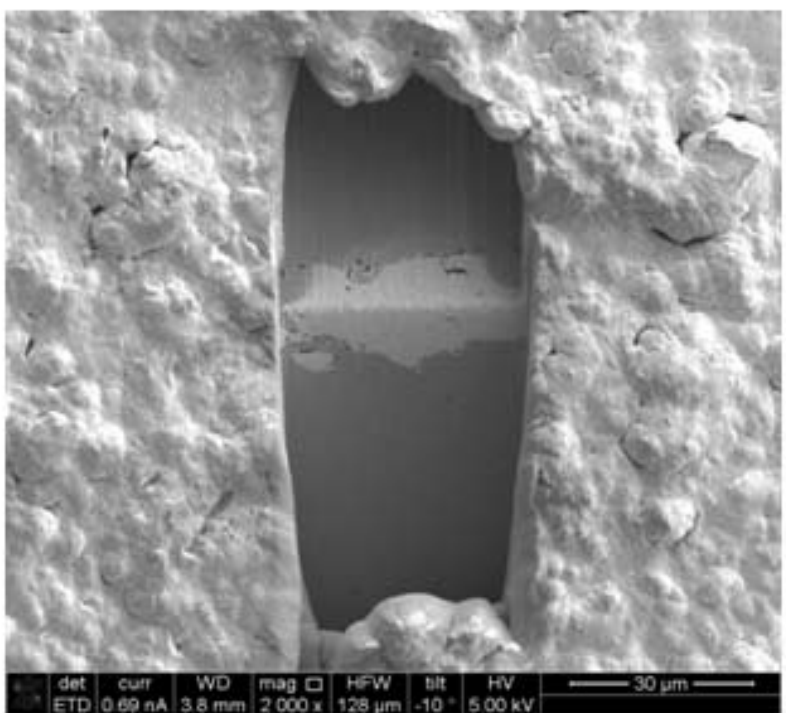

Figure 2. SEM image of cross section after milling from both orientations.

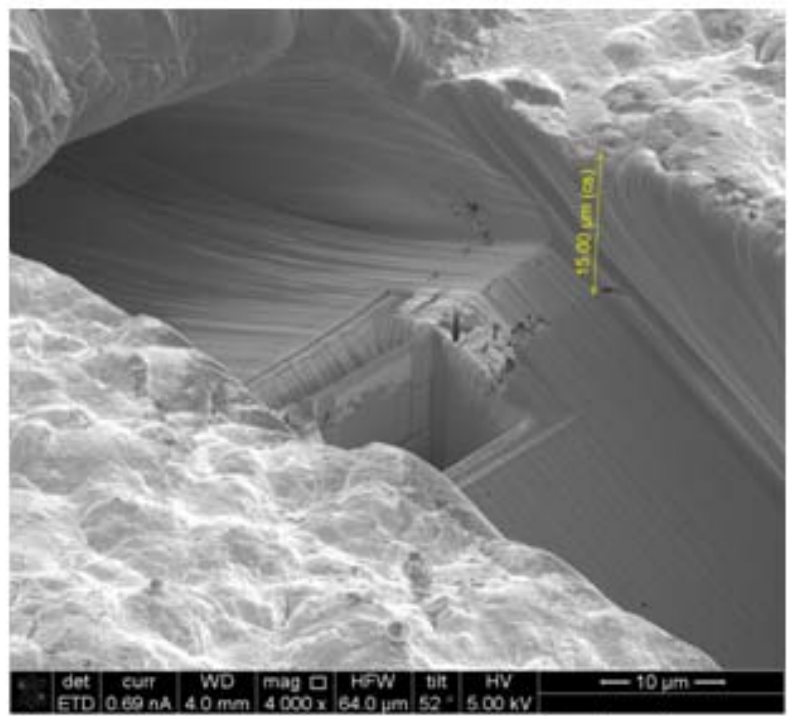

Figure 4. SEM image of TEM cross section showing the buried interface

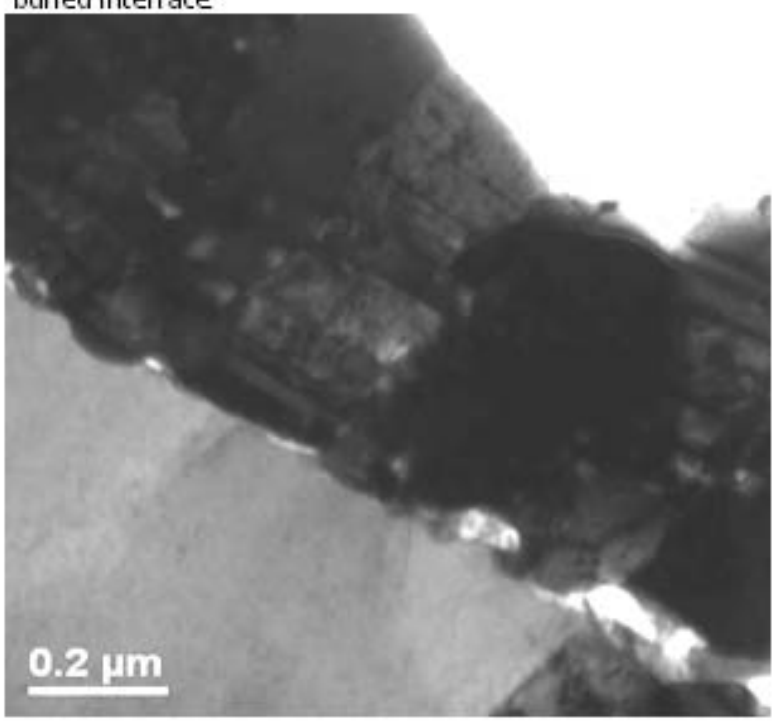

Figure6. TEM image showing the buried interface 